\title{
Prolactinoma: un diagnóstico que debe tenerse en cuenta en niños con neoplasias hipofisarias
}

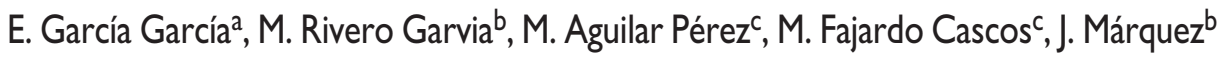 \\ anidad de Endocrinología Pediátrica. \\ 'Servicio de Neurocirugía. \\ 'Servicio de Radiodiagnóstico. \\ Hospitales Universitarios Virgen del Rocío. Sevilla. España.
}

Rev Pediatr Aten Primaria. 2009; I I:607-12

Emilio García García, ggej@hotmail.com

Resumen

En la infancia y la adolescencia, los adenomas hipofisarios son raros, y la mitad de ellos son prolactinomas. Se presenta un niño de 13 años con una lesión quística selar y supraselar tratada con cirugía, tras la cual el examen histológico e inmunohistoquímico diagnosticó un prolactinoma. Los macroprolactinomas quísticos antes de la pubertad son muy raros, pero se han descrito desde la edad de 9 años. El diagnóstico diferencial con el craneofaringioma quístico es muy importante, puesto que el tratamiento de primera elección del prolactinoma son los agonistas dopaminérgicos, mientras que en el craneofaringioma se prefiere la cirugía transcraneal.

Palabras clave: Prolactinoma, Neoplasias hipofisarias, Apoplejía hipofisaria, Niño, Adolescente.

\section{Abstract}

Pituitary adenomas are rare in childhood and adolescence and half of them are prolactinomas. In this report, we present a 13-year-old boy with a sellar and suprasellar cystic lesion treated with surgery; afterwards, histopathological and immunohistochemical examinations revealed a prolactinoma. Cystic macroprolactinomas are extremely rare in prepuberty, nevertheless they have been reported from the age of 9 years. The differential diagnosis with cystic craniopharyngioma is important, as treatment with dopamine-agonists may be the first choice in prolatinomas, whereas a transcranial surgery is required for a suprasellar craniopharyngioma.

Key words: Prolactinoma, Pituitary neoplasms, Pituitary apoplexy, Child, Adolescent.

\section{Introducción}

El prolactinoma es el tumor hipofisario más frecuente en adultos; sin embargo, en la edad pediátrica es raro. En las series más largas publicadas, el prolactinoma es el segundo adenoma hipofisario en la infancia, tras el productor de corticotropina $(\mathrm{ACTH})$, y el más fre-

Los autores declaran no presentar conflictos de intereses en relación con la preparación y publicación de este artículo. 
cuente en la adolescencia; predomina en mujeres mayores de 12 años ${ }^{1-10}$.

Se han descrito casos de prolactinoma en niños prepúberes en los que alcanza un tamaño gigante y se hace quístico por hemorragia intratumoral, adquiriendo un aspecto radiológico parecido al del craneofaringioma, tumor de la región hipofisaria más frecuente en las edades pediátricas ${ }^{11,12}$.

\section{Caso clínico}

Varón de 13 años sin antecedentes de interés que consultó por cefalea frontal y nicturia en los meses previos.
En la exploración presentó talla y peso normales para su edad, ginecomastia bilateral de $2 \mathrm{~cm}$ de diámetro, pubarquia y testes de $3 \mathrm{ml}$. La exploración oftalmológica era normal.

La tomografía computarizada (TC) descubrió un proceso expansivo intraselar (emergía desde el interior de la silla turca) con crecimiento supraselar, bien delimitado, de unos $3 \mathrm{~cm}$ de diámetro máximo, isodenso respecto al parénquima, de aspecto quístico con polo sólido y anillo periférico hipercaptantes tras la inyección de contraste (figura 1). La resonancia magnética (RM)

Figura 1. Tomografía computarizada con contraste. Corte axial que muestra un proceso expansivo intra-supraselar con la zona posterior ligeramente hipercaptante y un intenso realce periférico.

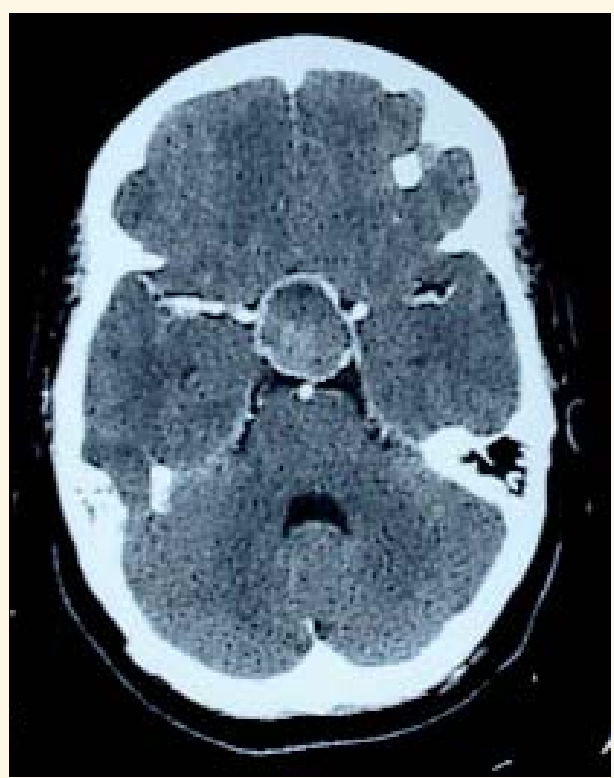


definió la porción quística supraselar de contenido proteináceo (ligeramente hiperintensa en T1 e hipointensa en T2) y la porción sólida intraselar isointensa a la sustancia gris en ambas secuencias y que realzaba intensamente tras gadolinio (figuras 2 y 3 ). Con el diagnóstico radiológico de craneofaringioma se indicó un tratamiento quirúrgico (no se había determinado el nivel de prolactina), y por abordaje transciliar derecho se realizó una exéresis macroscópicamente completa. La anatomía patológica diagnosticó adenoma hipofisario con inmunohistoquímica positiva para prolactina y cromogranina A.

Tras la intervención, el paciente presentó una hemianopsia bitemporal con extensión mayor en el campo visual derecho y panhipopituitarismo, y fue derivado a endocrinología. Los niveles de prolactina tras la intervención eran de $83 \mathrm{ng} / \mathrm{ml}$, sin restos del adenoma en la $\mathrm{RM}$, y se inició tratamiento con cabergolina. La calcemia era normal $(9,5$ y $8,8 \mathrm{mg} / \mathrm{dl}$ ), por lo que no existe sospecha de hiperparatiroidismo ni de otros tumores propios de la neoplasia endocrina múltiple (MEN 1).

Figura 2. Resonancia magnética. Corte sagital que muestra cómo la lesión se origina en el interior de la silla turca y se extiende supraselarmente por encima del diafragma. No se identifica hipófisis normal.

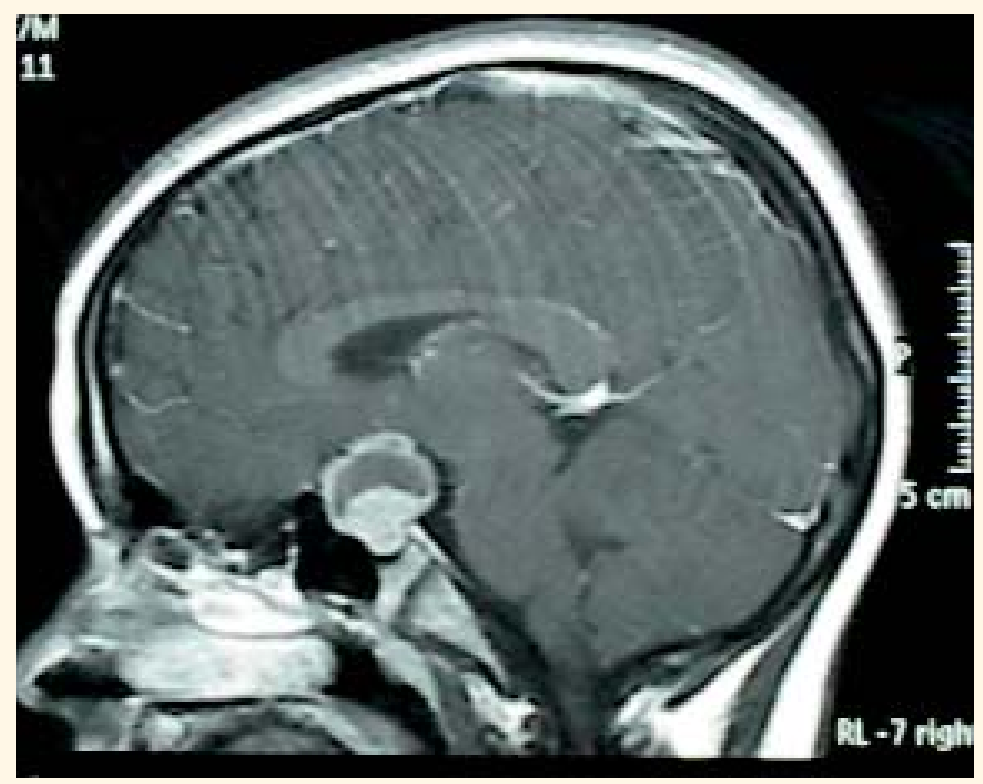




\section{Discusión}

El diagnóstico diferencial de un tumor selar o paraselar en un niño incluye, como primera posibilidad, el craneofaringioma, por ser el más frecuente en las dos primeras décadas de la vida, sobre todo si la masa es quística y presenta calcificaciones. Además, el craneofaringioma puede tener un origen intraselar, como el adenoma hipofisario. Otras lesiones de esta área incluyen el tumor de células germinales, el quiste de la bolsa de Rathke, los tumores dermoides y epidermoides, los meningiomas (que también pueden calcificarse) y los gliomas de las vías ópticas. En las lesiones extrahipofisarias, la RM puede identificar la hipófisis rechazada o comprimida, pero no en el adenoma ${ }^{1-10}$.

En las series de menores de 20 años, el prolactinoma es más frecuente en la mujer con una relación $3: 1^{3,13}$. En la mujer pospuberal suele diagnosticarse precozmente, cuando aún es microprolactinoma, por las manifestaciones típicas de la hiperprolactinemia (galactorrea y amenorrea). Sin embargo, en la mujer antes de la pubertad y en el varón se suele diagnosticar tardíamente, cuando ya es macroadenoma ${ }^{3,5,13}$. Las formas de pre-

Figura 3. Resonancia magnética con gadolinio. Corte coronal que muestra cómo el polo sólido intraselar se realza intensamente.

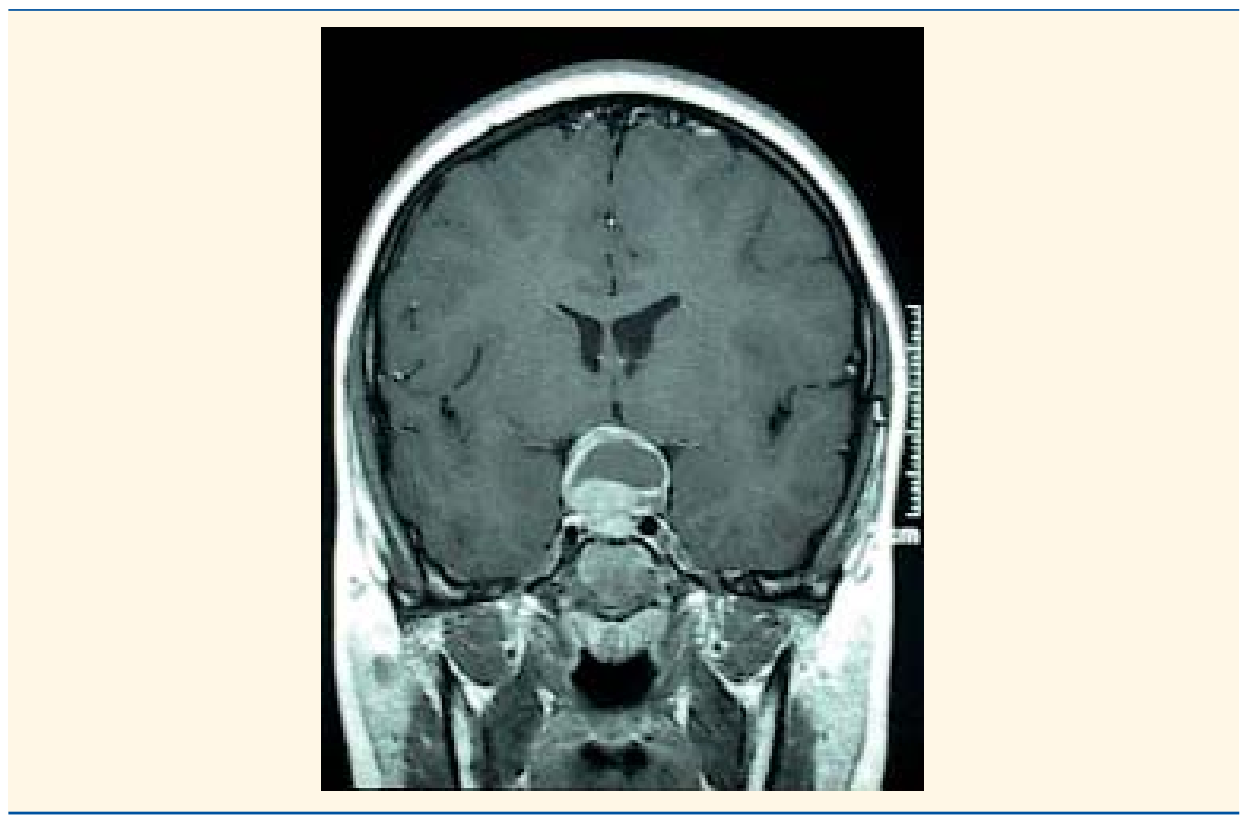


sentación más frecuentes en estos casos son la cefalea y los defectos visuales, dado que las manifestaciones endocrinológicas (retraso puberal, ginecomastia en el varón) suelen pasar desapercibidas ${ }^{2,5}$. Se ha descrito algún caso de galactorrea en el varón, incluso en un niño de 8 años ${ }^{14}$, pero no es un signo frecuente 2 .

Los macroprolactinomas pueden producir apoplejía hipofisaria como forma de debut clínico en la infancia (cuadro brusco de hipertensión craneal y déficit visual). Estos cambios degenerativos, necróticos y hemorrágicos de una parte del adenoma le darán aspecto quístico fácil de confundir con otras lesiones ${ }^{15}$.

Algunos autores piensan que el prolactinoma en la edad pediátrica es más invasivo ${ }^{16}$; sin embargo, otros no están de acuerdo ${ }^{1,4,7,9}$. Se ha publicado un caso maligno en un niño de 12 años con metástasis craneoespinales 16 meses después de la extirpación del tumor primario ${ }^{17}$.

Como en los adultos, los agonistas dopaminérgicos son la primera elec- ción en el tratamiento del prolactino$\mathrm{ma}^{2,3,18}$, ya que consiguen una disminución significativa del tamaño en más de la mitad de los casos, normalizan el nivel de prolactina con restauración de la función gonadal en el $80 \%$ de los pacientes y preservan el resto de la función anterohipofisaria ${ }^{2,3,19}$. La cabergolina consigue respuesta en el $70 \%$ de los casos resistentes a otros agonistas ${ }^{2}$. Se ha publicado el caso de un niño de 7 años con un prolactinoma gigante en el que la cabergolina normalizó la prolactina en 6 semanas y redujo el tamaño tumoral, mejorando el exoftalmos, la anisocoria y el déficit visual que producía ${ }^{11}$. La cirugía queda, pues, reservada para los casos de intolerancia o resistencia a la medicación ${ }^{13}$.

Puesto que el prolactinoma puede beneficiarse de tratamiento médico, ante cualquier masa selar o periselar -también en la edad pediátrica- debe medirse el nivel de prolactina antes de plantear el tratamiento quirúrgico.

\section{Bibliografía}

1. Partington MD, Davis DH, Laws ER Jr, Scheithauer BW. Pituitary adenomas in childhood and adolescence. Results of transsphenoidal surgery. J Neurosurg. 1994;80:209-16.
2. Colao A, Loche S, Cappa M. Prolactinomas in children and adolescents. Clinical presentation and long-term follow-up. J Clin Endocrinol Metab. 1998;83:2777-80.

3. Fideleff $H L$, Boquete $H R$, Sequera $A$, Suárez $M$, Sobrado P, Giaccio A. Peripubertal prolacti- 
nomas: clinical presentation and long-term outcome with different therapeutic approaches. J Pediatr Endocrinol Metab. 2000;13:261-7.

4. Maira G, Anile C. Pituitary adenomas in childhood and adolescence. Can J Neurol Sci. 1990;17:83-7.

5. Mindermann T, Wilson CB. Pediatric pituitary adenomas. Neurosurgery. 1995;36:259-68.

6. Kunwar S, Wilson CB. Pediatric pituitary adenomas. J Clin Endocrinol Metab. 1999;84: 4385-9.

7. Pandey P, Ojha BK, Mahapatra AK. Pediatric pituitary adenoma: a serie of 42 patients. J Clin Neurosci. 2005;12:124-7.

8. De Menis E, Visentin A, Billeci D. Pituitary adenomas in childhood and adolescence. Clinical analysis of 10 cases. J Endocrinol Invest. 2001;24: 92-7.

9. Kane LA, Leinung MC, Scheithauer BW. Pituitary adenomas in childhood and adolescence. J Clin Endocrinol Metab. 1994;79:1135-40.

10. Webb C, Prayson RA. Pediatric pituitary adenomas. Arch Pathol Lab Med. 2008;132:77-80.

11. Krassas GE, Pontikides N, Kaltsas T. Giant prolactinoma presented as unilateral exophthalmos in a prepubertal boy: response to cabergoline. Horm Res. 1999;52:45-8.

12. Dinc C, Bikmaz K, Iplikcioglu AC, Kosdere $\mathrm{S}$, Latifaci I. Cystic giant prolactinoma in childhood. J Clin Neurosci. 2008;15:76-9.
13. Abe T, Lüdecke DK. Transnasal surgery for prolactin-secreting pituitary adenomas in childhood and adolescence. Surg Neurol. 2002;57: 369-78.

14. Ross RJ, McEniery JM, Grossman A, Doniach I, Besser GM, Savage MO. Massive prolactinoma with galactorrhoea in a prepubertal boy. Postgrad Med J. 1989;65:403-6.

15. Yang $M H$, Chuang $H$, Jung $S M$, Van $Y H_{\text {, }}$ Lo FS. Pituitary apoplexy due to prolactinoma in a Taiwanese boy: patient report and review of the literature. J Pediatr Endocrinol Metab. 2003; 16:1301-5.

16. Kanter SL, Mickle JP, Hunter SB, Rhoton AL. Pituitary adenomas in pediatric patients: are they more invasive? Pediatr Neurosci. 19851986;12:202-4.

17. Huang $A P$, Yang $S H$, Yang $C C$, Kuo MF, Wu MZ, Tu YK. Malignant prolactinoma with craniospinal metastasis in a 12-year-old boy. J Neurooncol. 2008;90:41-6.

18. Duntas LH. Prolactinomas in children and adolescents: consequences in adult life. J Pediatr Endocrinol Metab. 2001;14 Suppl 5:1227-32.

19. Sakazume S, Obata K, Takahashi E. Bromocriptine treatment of prolactinoma restores growth hormone secretion and causes catch-up growth in a prepubertal child. Eur J Pediatr. 2004;163:472-4. 